\title{
Riesgos ambientales y desarrollo en la zona rural
}

Salvador Orlando Alfaro

Universidad de Regina

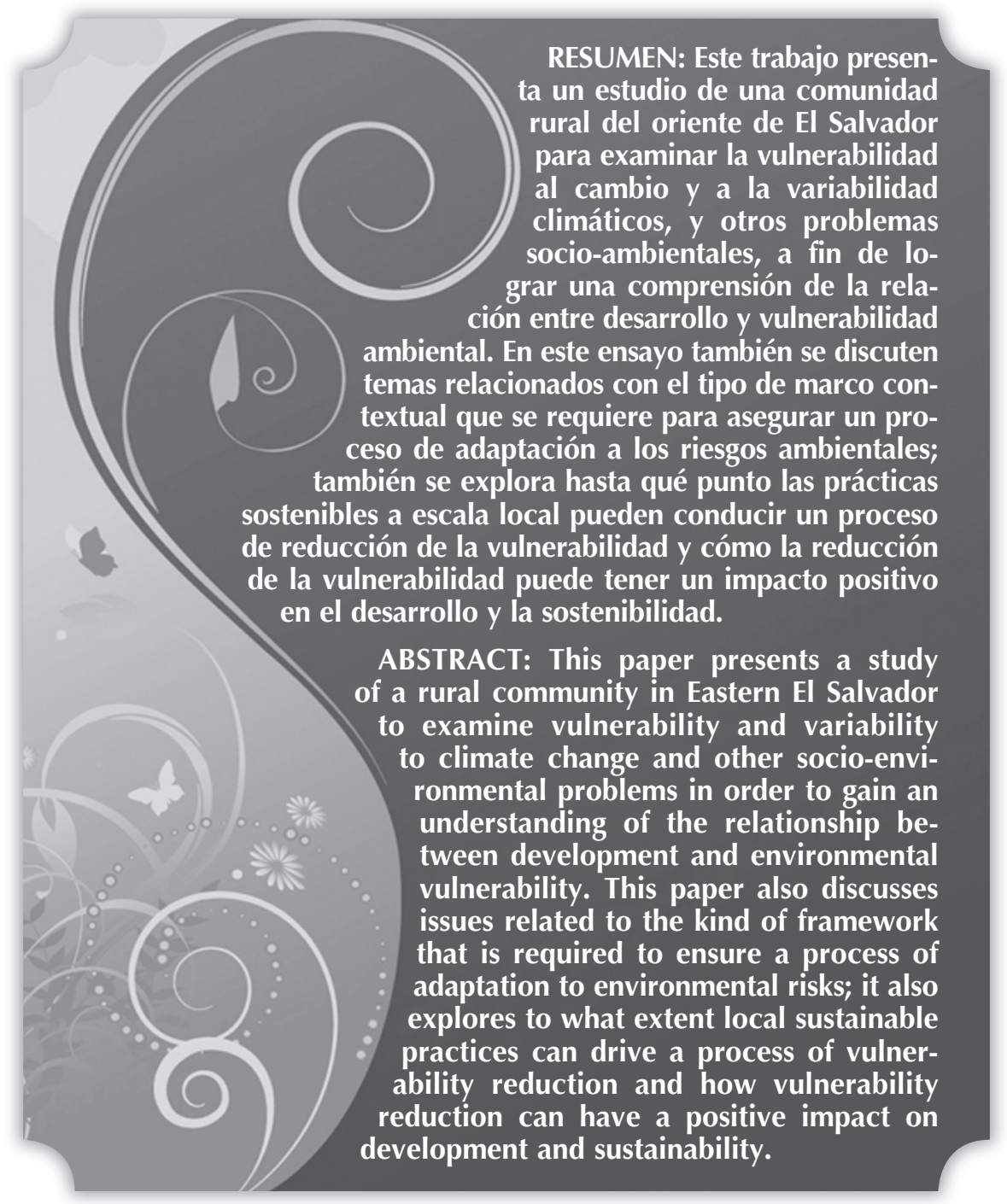


E ste trabajo presenta una discusión de los procesos que tienen lugar en una comunidad rural del oriente de El Salvador en relación con su vulnerabilidad al cambio y a la variabilidad climáticos, a fin de lograr una mejor comprensión de la relación entre el desarrollo y los desastres, y acerca de qué tipo de marco contextual se requiere para garantizar un proceso de adaptación a los riesgos y a las dinámicas de riesgo. Este trabajo explora en qué medida el desarrollo puede conducir a un proceso de reducción de la vulnerabilidad y viceversa. Indica que las percepciones de riesgo y vulnerabilidad juegan un papel fundamental en determinar cuán bajo riesgo se encuentra la población salvadoreña actualmente. Esto tiene implicaciones significativas para el proceso de desarrollo, porque las percepciones pueden impedirle a los grupos e individuos que apoyen la reducción de la pobreza, las prácticas sostenibles y las estrategias de empoderamiento local. Esta discusión también indica que los factores que inicialmente pudieron haber parecido importantes para determinar el riesgo, esto es, los actuales riesgos del cambio climático, tienen tan sólo un papel menor que jugar a la hora de determinar el riesgo. Por tanto, la integración de los temas de riesgo climático dentro del desarrollo no debe simplemente etiquetarse bajo la rúbrica de la incertidumbre científica sobre el cambio climático: el papel del riesgo climático en la obstaculización del desarrollo debe enfrentarse ya, porque ya es un elemento de primera importancia. Los factores que restringen la reducción de la vulnerabilidad y el desarrollo están claramente relacionados y suelen ser idénticos.

El grado en el que los individuos o las sociedades son capaces de permanecer incólumes ante el cambio y la variabilidad climáticos no se basa solamente en las tecnologías y el conocimiento que se poseen. Como puede verse en $\mathrm{El}$ Salvador, la manera en que los impactos de la vulnerabilidad climática se experimentan se ve determinada por un amplio espectro de factores socio-económicos y culturales relacionados con la pobreza, el empleo, las creencias ideológicas, la dependencia, las instituciones y los conocimientos disponibles sobre el gestión de riesgos. Por lo tanto, en El Salvador parece que la variabilidad climática es algo de menor importancia para determinar los riesgos, si se le compara con aquellos factores que causan vulnerabilidad. Esto se basa en la comprensión de que el riesgo es el producto de la interacción entre el peligro y la vulnerabilidad a dicho peligro, representada por la ecuación conceptual Riesgo $=$ Peligro + vulnerabilidad (Wisner et al. 2004) 
Aunque la vulnerabilidad climática es algo inherente a la sociedad salvadoreña, esto no garantiza que las personas en cuestión estén bien adaptadas a su medio ambiente. Puesto que es relativamente cierto que los riesgos climáticos se incrementarán en frecuencia o magnitud, o ambos, como resultado del cambio climático, los riesgos para los pobladores en el caso de esta comunidad rural en particular pueden reducirse solamente si el aumento en el riesgo se ve más que recompensado por una reducción en la vulnerabilidad a dichos riesgos. Es en el terreno de las opciones relativas a sus medios de vida en donde los individuos y las comunidades pueden tener los mayores efectos en su vulnerabilidad. En este sentido, estos retos indican que para perseguir la adaptación a la variabilidad del cambio climático, debe echarse mano de una perspectiva integral e incluyente, con lo cual se toman en cuenta las características de las estructuras de gobernanza nacional. Para promover un proceso de adaptación en El Salvador, será necesario enfrentar numerosos asuntos, incluyendo la dinámica económica estructural, así como temas de percepción. Este texto discute esos asuntos críticos.

\section{II}

Ubicado en la parte oriental del país, el Bajo Lempa está rodeado por la costa del Pacífico y vincula, por medio de carretera, a la capital del país con la zona oriental de Honduras. Está determinado por el río Lempa, que parte la zona en dos. La carretera recorre el único puente que une a dichos territorios. El área tiene aproximadamente 807 kms. $^{2}$ (Medina et al., 2002) y se caracteriza como una zona a nivel del mar que queda inundada con la crecida del río. Se calcula la población entre 30 y 40,000 personas que viven en unas noventa comunidades, muchas de las cuales son extremadamente pobres. Muchas de estas comunidades ocuparon la zona después de 1992, tras la firma de los acuerdos de paz entre el gobierno y el FMLN. Una parte de dichos acuerdos incluía el reparto de tierra en el valle del Bajo Lempa para los miembros de las fuerzas anteriormente enfrentadas. Se construyeron nuevos asentamientos en la zona a la par de asentamientos previamente construidos y muchos de ellos estaban ubicados en zonas proclives a las inundaciones. Una parte significativa de esta población inmigrante provenía de tierras altas e incluso de zonas urbanas y tenían muy poca experiencia sobre las condiciones de vida en tierras bajas que se ven sometidas a inundaciones.

Poca gente vivía en el Bajo Lempa antes de la guerra, dada la alta prevalencia del dengue y la 
malaria y había, por tanto, pocos registros de "desastres". Sin embargo, el área era conocida como el "granero" de El Salvador hasta finales de los 70, dada su alta productividad de sus industrias algodoneras y azucareras (Cuéllar et al., 2002). Durante el conflicto armado, sin embargo, la región servía como zona de combate y se le consideraba muy peligrosa, de tal suerte que muchos habitantes que habían sido empleados en las haciendas decidieron evacuar. Muchos de los latifundios se rompieron a raíz de la reforma agraria de 1980 (Lavell 2004) y después de que la Constitución de 1983 estableciera un techo de 245 hectáreas en la cantidad de tierra que podía poseer un individuo (Brockett 1990).

Desde 1992, la zona ha sufrido muchos incidentes de inundaciones y, más recientemente, el impacto de los terremotos de 2001. La inundación asociada con el huracán Mitch de 1998 fue particularmente dramática luego de que la apertura de las compuertas de la presa "15 de septiembre" superó los niveles de inundación previstos. Los impactos de las inundaciones han sido severos en la zona desde 1992, debido, principalmente, a los altos niveles de vulnerabilidad y a los bajos niveles de recuperación de la población, a la falta de una infraestructura de protección física adecuada y a los patrones de inundación cambiantes debido al cambio ambiental y al impacto de las estructuras de la presa hidroeléctrica.

Aunque Walker y Jodha apuntan que, en general, "los agricultores de las zonas agrícolas riesgosas han desarrollado diversas medidas para enfrentar los riesgos productivos" (1986: 18), los habitantes del Bajo Lempa no se habían visto expuestos a las condiciones presentes durante más de once años, dado que los primeros pobladores llegaron a fines de 1991 y a principios del año siguiente. En general, parece que los habitantes del Bajo Lempa no tenían suficiente experiencia en las condiciones ambientales para los cultivos (Medina et al. 2002). Algunas personas provenían de áreas urbanas y no tenían ninguna experiencia de trabajo agrícola (Lavell 2004). Aquellas personas que no están familizarizadas con el ambiente de las riberas depositan su confianza en el gobierno salvadoreño y en las ONG para controlar físicamente al río Lempa, aunque la dinámica del río es tal, que se inunda con regularidad. Esto sugiere que los pobladores del Bajo Lempa están en un proceso de adaptación a las nuevas condiciones, presentando, por tanto, una ubicación ideal para estudiar la adaptación al cambio climático. 
El Salvador es uno de los países con recursos más reducidos y con un medio ambiente más dañado en América Latina (Acevedo et al. 1995), así como el más densamente poblado. Su alta tasa de natalidad y su pequeño territorio añaden mayor presión al medio ambiente y a los ecosistemas naturales, así como en los aspectos económicos y sociales de la vida, afectando las condiciones de vida rurales. De hecho, las condiciones estructurales generadas por las particularidades del capitalismo dependiente son el factor principal que explica las actuales relaciones sociales y medioambientales del país. Más aún, la vulnerabilidad social y física a los riesgos naturales, numerosos y frecuentes, que incluyen inundaciones, sequías, huracanes y eventos asociados a los anteriores, como deslizamientos de tierra, son significativos en diferentes regiones del país y, como consecuencia de ello, muchos salvadoreños enfrentan altos riesgos todos los días.

Algunos estudios muestran que la interpretación del concepto de "gestión de riesgos" varía considerablemente en El Salvador e incluso las políticas que ponen el énfasis en medidas preventivas probablemente dejen intactas aquellas concepciones erradas tradicionalmente aceptadas, las cuales atribuyen los desastres a la naturaleza, con lo cual, la actitud derrotista derivada de lo anterior se vuelve sumamente evidente. Similarmente, pese a los cambios institucionales en el monitoreo de riesgo, que aparece ser relativamente exitoso en lo tocante a la comunicación y a la concientización, el conflicto armado que terminó en 1992 dejó una herencia de desconfianza política hacia el régimen de tenencia de la tierra y otros temas, trajeron consigo altas tasas de desempleo y contribuyeron a la marginación del sector agrícola. Mientras que el sector agrícola es sumamente sensible a la variabilidad del clima, la evidencia de campo indica que este es uno de los muchos factores que contribuyen a su deterioro y declive. Esto se debe a que las causas radicales de la vulnerabilidad social en El Salvador son el resultado de las particularidades del capitalismo dependiente, su modelo de desarrollo actual y la historia de violencia política, incluyendo la subordinación a las políticas agrícolas y geopolíticas estadounidenses.

En este contexto, el análisis de la respuesta de la población local al cambio climático en el Bajo Lempa demuestra claramente que hay un alto grado de conciencia acerca del riesgo que suponen tanto las inundaciones como la sequía en otro tipo de fenómenos físicos. Sin embargo, hay niveles variables de interés en tomar medidas para reducir los impactos. Las razones abarcan 
desde las creencias culturales hasta temas de dependencia.

Los hallazgos que identificaron Moisa (1996) y Perla-Alfaro (2006) indican que hay diferentes tipos de respuesta en los niveles individuales y comunitarios. Mientras que la respuesta a las inundaciones y sequías aparecen asociadas con la organización en un nivel comunitario, a nivel individual se pudo identificar una variedad mayor de opciones.

Por consiguiente, la mayoría de las respuestas individuales están relacionadas con la práctica agrícola y a las decisiones individuales sobre cómo organizar la temporada de cultivo, incluyendo cuándo comenzar la siembra. Hay otras respuestas que están vinculadas claramente a problemas de pobreza; en particular, la migración se plantea como una opción frecuente para la sociedad salvadoreña, a fin de intentar escapar a la pobreza y el desempleo. La pobreza en la organización de afuera es otra forma de respuesta a las condiciones climáticas inciertas. Talvez uno de los problemas principales que se asocian con esta dependencia es el sentimiento de victimización relacionado con la ayuda para las emergencias que también podría actuar como una barrera para el propio empoderamiento, limitando las capacidades de la gente para identificar oportunidades de empleo y sobrevivencia. Aunque la actitud de los excombatientes es evidente entre los habitantes del Bajo Lempa, lo cual refuerza el punto de vista de que es posible cumplir objetivos a través de la persistencia y la dedicación, ya sea por la lucha violenta o pacífica, la frustración sobre el riesgo constante que presentan la pobreza, las inundaciones y las sequías inmediatamente después de doce años de conflicto armado puede ser suficientes para que los individuos se sientan como víctimas. Por consiguiente, las actitudes y la motivación parecen jugar un papel importante en el proceso de adaptación a las nuevas condiciones climáticas.

Aunque claramente hay capacidad a nivel local en el Bajo Lempa, relacionada principalmente a las redes y organizaciones sociales, las estrategias o políticas nacionales o regionales en conflicto puede anular los planes y decisiones a nivel local. En el contexto del cambio climático y el desarrollo, la agricultura es un sector clave, donde la variabilidad en el clima se traduce en el impacto en la alimentación, la forma de vida, el ingreso y la seguridad alimentaria. La evidencia que proviene del Bajo Lempa indica que los agricultores están haciendo ajustes a la temporada de cultivos; sin embargo, la falta de mercados para sus productos y el Tratado de Libre Comercio con Centroamérica (CAFTA, por sus siglas en inglés) con los Estados Unidos tendrá consecuencias para las prácticas agrícolas de los productores rurales. Como ya se 
anotó anteriormente, la vulnerabilidad agrícola se ve determinada por numerosos factores, pero parece que el clima no es el factor de riesgo más decisivo para los habitantes del Bajo Lempa. Hay otros factores que juegan un papel mayor a la hora de determinar la vulnerabilidad, entre ellos, por ejemplo, se encuentra el estancamiento en el sector agrícola y en la economía rural, la desconfianza en las instituciones gubernamentales, la dependencia en el sector de las ONG, las estructuras gubernamentales para el gestión de riesgos y las diferentes comprensiones y creencias. Estos factores afectan la capacidad de los habitantes para llevar a cabo respuestas adecuadas para los riesgos ambientales, incluyendo las políticas y actitudes hacia los riesgos y la vulnerabilidad producidos por las inundaciones y las sequías, que se evidencia en El Salvador. Importancia particular reviste la forma como el manejo de los desastres se define de cara al manejo de los riesgos, y el papel e impacto de las agencias de asistencia, principalmente las ONG y las organizaciones de ayuda internacional, para enfrentar la vulnerabilidad, el riesgo y el desastre. Entonces, por ejemplo, las estructuras gubernamentales de gestión de riesgos hacen un énfasis mayor en la asistencia en caso de desastres que en las medidas de precaución, confundiendo así la gestión de riesgos con el manejo de desastres.

\section{IV}

La gestión de riesgos suele definirse actualmente como las intervenciones orientadas a reducir la vulnerabilidad a los peligros, dado que hay muchos riesgos que no pueden controlarse, sino tan solo evitarse y con la comprensión de que es la vulnerabilidad humana a dichos peligros es lo que genera riesgos (Wisner et al. 2004). El interés académico reciente en la vulnerabilidad ha influido claramente en el pensamiento sobre el tema en El Salvador. El que los desastres no son naturales es una noción consensuada entre ciertos sectores interesados en el tema en El Salvador. Sin embargo, el manejo de desastres implementado por el gobierno salvadoreño y otras organizaciones parece desconocer esto por completo y la evidencia sugiere que todavía existe una perspectiva no integrada con respecto a la ayuda para los desastres y el gestión de riesgos en el país. El manejo de los desastres se usa comúnmente para describir las actividades que siguen después de un desastre, pero el gestión de riesgos incorpora acciones preventivas y se enfoca en un panorama más amplio de las causas. Definir la gestión de riesgos como una forma más integrada, abarcadora y sostenible de manejo de desastres en El Salvador sigue siendo un desafío. En el país parece darse cierta representación errada 
del significado de los conceptos entre todos los sectores interesados al respecto. Algunos de ellos ven la ayuda al desastre como una forma de adaptarse al cambio climático - lo cual se asocia normalmente con un ajuste a largo plazo y sostenible a los cambios, en vez de asociarlo con una respuesta inmediata a los eventos extremos. Los hallazgos indican que la confianza depositada en la ayuda externa, tanto de parte del gobierno como de los habitantes de las comunidades y, más aún, la confianza en la ayuda como una estrategia de enfrentar los desastres puede ser insostenible y producir una mala adaptación. La evidencia muestra que, al menos en parte, la falta de preparación para responder a las inundaciones y la sequía en El Salvador se basa en la creencia en que el no tomar acciones resultará en la ayuda externa en forma de asistencia para los desastres y que, dado lo anterior, el gobierno no tiene que responsabilizarse para enfrentar los costos de la reconstrucción.

En relación con lo que conlleva la gestión de riesgos, hay evidencia de que el gobierno salvadoreño $-\mathrm{y}$, posiblemente, otras agencias que juegan un papel importante en la preparación y las condiciones existentes para dar respuesta a los desastres - han usado mal el concepto. Es posible que los conflictos entre Protección Civil y el Servicio Nacional de Estudios Territoriales (SNET) —una entidad que abarca unidades de meteorología, hidrología, sismología y gestión de riesgos- puede basarse en formas contrastadas de entender el gestión de riesgos. La definición del concepto no tiene solamente consecuencias para la planificación, sino que también implica la cuestión de qué tipo de manejo se lleva a cabo en El Salvador. Wisner ha notado que "si el desarrollo lleva a la reducción de desastres, esto depende del tipo de 'desarrollo'" (2001: 261). Este punto de vista ha tenido eco en un comentario hecho por un miembro de la comunidad: "Yo no tengo confianza [en el SNET]. ¿Cual es su objetivo? Si no cuestionan [el modelo de desarroIlo en El Salvador] no van a poder enfrentar el gestión de riesgos". De nuevo, lo organizativo y la presencia de organizaciones preocupadas por los riesgos se enfatizan a nivel nacional como una característica de un "buen nivel de preparación". De forma similar, se considera que la prueba de la organización reside en saber qué hacer a la hora de una emergencia. Por esta razón, los mapas de riesgo se destacan por cuanto están destinados a informar a la población rural sobre el lugar a donde deben ir para exponerse al menor nivel de riesgo, por ejemplo, en el caso de una inundación. La idea de un mapa de riesgos se toma como parte del gestión de riesgos, aunque, al parecer, la gente de las comunidades no sabe cómo usar dichos mapas. Los mapas de riesgo tienen el potencial de crear un 
falso sentimiento de seguridad en las áreas más proclives a riesgos. Es claro que una comprensión común de lo que abarca el gestión de riesgos sería muy útil a fin de que las políticas y los esfuerzos se complementen mutuamente y que también se empleen de manera efectiva.

Según las definiciones encontradas en el sitio de internet del SNET, el concepto de gestión de riesgos se entiende como un "proceso social complejo que conduce al planeamiento y aplicación de políticas, estrategias, instrumentos y medidas orientadas a impedir, reducir, prever y controlar los efectos adversos de fenómenos peligrosos sobre la población, los bienes y servicios y el ambiente" y como las "acciones integradas de reducción de riesgos a través de actividades de prevención, mitigación, preparación para, y atención de emergencias y recuperación post impacto" (Sitio de internet de SNET, 2006). Nótese que la última definición incluye, además, las actividades posteriores al evento. El desastre se define como una "situación o proceso social que se desencadena como resultado de la manifestación de un fenómeno de origen natural, tecnológico o provocado por el hombre que, al encontrar condiciones propicias de vulnerabilidad en una población, causa alteraciones intensas, graves y extendidas en las condiciones normales de funcionamiento de la comunidad". El SNET está consciente de la diferencia entre la filosofía tradicional consistente en una comprensión de la amenaza centrada en el riesgo y la "nueva" filosofía del manejo de riesgo enfocada en la vulnerabilidad. El SNET rechaza el viejo punto de vista, según el cual el manejo de riesgo se equipara a la atención en caso de emergencia La Dirección General de Protección Civil, una dependencia gubernamental creada en la presente administración para sustituir al viejo Comité de Emergencia Nacional, COEN, define el término amenaza como un "peligro latente que representa la posible manifestación dentro de un período de tiempo y en un territorio particular de un fenómeno de origen natural, socio-natural o antrópogenico, que puede producir efectos adversos en las personas, la producción, la infraestructura, los bienes y servicios y el ambiente. Es un factor de riesgo externo de un elemento o grupo de elementos expuestos, que se expresa como la probabilidad de que un evento se presente con una cierta intensidad, en un sitio especifico y dentro de un periodo de tiempo definido" (Sitio de internet de la Dirección General de Protección Civil 2009). En tanto sus tareas incluyen la atención de emergencias, se entiende la gestión de riesgos como manejo de desastres.

La coordinación y la colaboración entre corporaciones con capacidades diferentes también se consideran como una forma de gestión de riesgos también en El Salvador, 
pero también son evidentes algunos obstáculos para hacer esto más eficiente. El desafío mayor podría residir en tratar de superar los énfasis que difieren entre una perspectiva centralizada en medidas de precaución (SNET) con aquella que esta enfocada en la atención posterior al evento (DPC). Por tanto, la gestión de riesgos parece tener un enfoque mayor en la respuesta a la emergencia que en las medidas de prevención y esto se refleja en las perspectivas de las agencias de ayuda y en las ONG.
Como resultado de lo anterior, se nota que existe un "constante estado de emergencia en el país". Esta idea es potencialmente peligrosa en el sentido de que refuerza una actitud de autovictimización que también es patente a escala local e indica una causa psicológica de la vulnerabilidad. Con ello, se considera que algo así como un estado permanente de emergencia también demuestra ambigüedad en las metas de desarrollo y de reducción de la vulnerabilidad y de las amenazas.

\section{V}

Los temas discutidos líneas arriba se han presentado como un desafío para la reducción efectiva del riesgo en El Salvador, lo cual es un prerrequisito para el proceso de adaptación al cambio climático. Esto se debe a que la reducción de la vulnerabilidad - un factor clave de la reducción del riesgotambién es el requisito subyacente de la adaptación. El caso del Bajo Lempa indica que hay una fuerte correlación entre la reducción de la vulnerabilidad y el camino hacia el desarrollo sustentable. Por tanto, los principales retos del desarrollo - tales como la marginación del sector agrícola - también representan un desafío mayor para la reducción del riesgo. Sobre este punto, Wisner subraya que una forma de garantizar una preparación mayor de cara a los desastres es iniciar un debate nacional "sobre la naturaleza y trayectoria del desarrollo en $\mathrm{El}$
Salvador" (2001: 265). Los factores discutidos representan aquellos que son más evidentes y que parecen ser más importantes a la hora de afectar la capacidad adaptativa en El Salvador. Estos factores no parecen ser exclusivos de El Salvador y la evidencia sugiere que se pueden hallar circunstancias similares en otros países latinoamericanos (Eakin 2005, Liverman 1999).

En El Salvador, la recuperación de posguerra ha conducido a una fragmentación de las redes de apoyo y de sistemas de creencias que en otro contexto facilitaban la adaptación. Existe una percepción pública en particular, según la cual la recurrencia de los peligros naturales conducirá eventualmente a la gente a descubrir que la "situación necesita cambiarse", implicando con ello que las pérdidas provocarán eventualmente 
ajustes. De forma similar, Adger y Brooks (2003) han observado que mientras el impacto en el clima ha aumentado la vulnerabilidad en el medio de corto a mediano plazo, la incidencia de los peligros puede fomentar la adaptación. Sin embargo, es cuestionable esperar un proceso de adaptación en el Bajo Lempa, por cuanto los factores limitantes ponen en calidad de desafío hasta los cambios más simples en las condiciones de vida rural. En el ámbito de la macropolítica, el gobierno de El Salvador podría tener la necesidad de iniciar modificaciones políticas a fin de permitir que la adaptación autónoma tome lugar, lo cual podría contrarrestar a otras políticas gubernamentales de desarrollo económica y podrían, por consiguiente, ser indeseables. Bajo esta apariencia de políticas audaces aparecen características que son propicias para contribuir a la capacidad de adaptación, pero estas no pueden tener éxito sin que se les acompañe de un ajuste en las políticas. De momento, parece que muchas de estas estrategias se asocian con debilidades relacionadas que sirven de obstáculo para alcanzar ajustes sostenibles acordes a las circunstancias medioambientales.

La organización, entendida como una fortaleza, ha sido analizada en numerosos países y situaciones, en particular, en el contexto del capital social y como un rasgo positivo de los procesos de adaptación (Pelling 2003), pero es apro- piado cuestionarse en qué medida la organización es realmente un recurso con el cual se puede contar para responder al cambio climático y como un elemento que facilite la capacidad de adaptación. El rol positivo de las redes sociales y de las organizaciones que contribuye al fomento de las capacidades de la organización social está ligado a la responsabilidad de estas mismas organizaciones en la creación y el refuerzo de la dependencia de las estructuras formales y de los actores externos en el Bajo Lempa. Se sugiere también que el síndrome de dependencia es también un límite a la acción autónoma y puede relacionarse con la falta de responsabilidad por los impactos de los peligros, incluyendo desastres tales como el huracán Mitch e, incluso, los terremotos de 2001. Sumada a la desconfianza generalizada del gobierno salvadoreño expresada en muchas respuestas, la dependencia en la organización y ayuda externas, y la carencia general de preparación para atender inundaciones y sequías evoca un sentimiento de victimización que es evidente a nivel local y también a escala nacional. La identificación de determinados grupos vulnerables como "víctimas" — de parte de ellos mismos ("autovictimización") y por parte de otros- puede identificarse como una "limitación conceptual" hacia la reducción de la vulnerabilidad por parte de quienes formulan las políticas y de las agencias para el desarrollo. El uso del concepto 
como una característica, como en el enunciado "soy vulnerable", fomenta el proceso de autovictimización entre aquellos que se sienten marginados y les quita el acceso a las oportunidades de enfrentarse a las fuerzas que determinan su vulnerabilidad. La percepción de los habitantes del Bajo Lempa de su vulnerabilidad y de los riesgos a los que están expuestos también contribuye a la forma en que se entiende la gestión de riesgos. Hay profundas diferencias ideológicas que apuntalan estos problemas y se ven favorecidas por influencias poderosas que han estado presentes en El Salvador y en cualquier otro lugar desde principios del siglo XX. Esto, a su vez, está conectado con preguntas que describen a El Salvador como un lugar que se encuentra "en estado de emergencia permanente", expresión empleada muchas veces por parte de diferentes organizaciones locales.

Claramente, el sentimiento de victimización en el Bajo Lempa también se puede vincular con la evidente frustración en el seno de la comunidad, generada por el hecho de que a sus miembros se les dieron tierras a las que ellos consideran que el gobierno inunda de forma deliberada (Perla-Alfaro 2006). Una de las características principales de los habitantes del Bajo Lempa es que están amarrados emocionalmente a su tierra, como resultado del PTT —el proceso de distribución de tierras inscrito en los Acuerdos de Paz- y el conflicto social que continúa siendo una herencia de la guerra. Los primeros asentamientos se dieron en 1991, antes de que el proceso oficial de redistribución de tierras tomara lugar. Como se describió, el terreno se ve expuesto a numerosos peligros meteorológicos y geológicos, que ponen en riesgo a los habitantes, puesto que están mal preparados para enfrentar los impactos de dichos peligros. Los estudios han identificado que "la transformación en los niveles de consciencia del riesgo y la reducción de riesgos y una comprensión más profunda de los 'procesos' de construcción de riesgos se necesitan en el Bajo Lempa, especialmente porque sus habitantes son inmigrantes recientes $y$ tienen muy poca experiencia con el medioambiente y la agricultura de las tierras bajas (Lavell 2004:80). El contexto de los nuevos asentamientos es importante cuando se buscan respuestas a los peligros, porque las comunidades no están acostumbradas a las conidiciones de la tierra como resultado del hecho de no haber pasado sus vidas en tales circunstancias climáticas - esto incluye las condiciones para cultivar, así como los aspectos socioculturales de verse expuestos al peligro. Otra características importante es que las comunidades están formadas por familias de El Salvador y algunas de ellas han pasado la mayor parte de sus vidas en Honduras. Puesto que las familias no están familiarizadas a la combinación de peligros que 
implica habitar el Bajo Lempa, no tienen suficiente tiempo para desarrollar estrategias adecuadas para enfrentar estas condiciones. Un examen de sus prácticas indica que hay intentos a este respecto, pero sin un conocimiento de la situación específica basado en la experiencia de vivir en una planicie aluvial. Esto se caracteriza como un proceso de adaptación. Para algunos, esto se ve como una oportunidad para aprender y desarrollarse en una forma más apropiada (Medina et al. 2002). Pero las preguntas todavía siguen siendo relevantes en el sentido de si es apropiado para estas personas vivir en una planicie aluvial y si, tal como lo plantean las opciones sugeridas por las ONG, los sistemas de irrigación y los cultivos resistentes a las inundaciones son la respuesta para encontrar el sustento en el Bajo Lempa.

Debajo de estas propuestas subyace la pregunta sobre la adaptación a tales condiciones extremas, considerando que la evidencia apunta a que las zonas del Bajo Lempa que se encuentran directamente adyacentes al río, donde está ubicada la mayoría de las comunidades, han sido una planicie aluvial al menos desde principios de los noventa. Al contrario de un cultivo como el arroz, los cultivos que se siembran en el Bajo Lempa no dependen de las inundaciones, sino que se ven dañados por ellas, aunque las inundaciones también se asocian con el incre- mento de los nutrientes del suelo. La pertinencia y la viabilidad de la adaptación y las respuestas a esta situación se pueden cuestionar. Es relevante formularse la cuestión de la pertinencia de adaptarse a estas condiciones desde una perspectiva propia de la ingeniería (por ejemplo, si vale la pena invertir para construir infraestructura física, tal como casas de refugio) y también desde la perspectiva del desarrollo (por ejemplo, si en el Bajo Lempa los habitantes sacrificarán otros aspectos de sus condiciones de vida a fin de adaptarse a las condiciones climáticas). La viabilidad de dicha adaptación debe formularse estructuralmente (por ejemplo, a través de ajustes físicos a las viviendas y a otra infraestructura necesaria para las condiciones de vida) y también desde una perspectiva del desarrollo: ¿hasta qué medida es sostenible habitar en una planicie aluvial, sobre todo si esta no forma parte de las condiciones de vida tradicionales? La pregunta sobre si esto es deseable, puede producir claramente diferentes reacciones, dependiendo de quien la responda. Parece que sería deseable para los habitantes del Bajo Lempa poder adaptarse a la variabilidad climática, porque es la única tierra sobre la cual tienen derechos. Además, también resulta claro que otros factores, tales como las oportunidades de empleo y las luchas políticas se arrogan una prioridad mayor. Ello, visto desde la perspectiva según la cual la reducción de la vulnerabili- 
dad necesita superar los principales desafíos del desarrollo; sin embargo, dichos factores deberían incluirse.

La agricultura es sensible a las variaciones en el clima (Bhandari 2003, Jones y Thornton 2003) y ha sido un indicador importante del cambio climático para los científicos naturales y sociales (IPCC 2001). Dos variables claves que se examinaron para valuar los impactos del cambio climático son los recursos hídricos y la seguridad alimentaria (Watson et al., 1998), los cuales se consideran como un tema clave en el desarrollo. La agricultura también es sensible a los impactos de la globalización, fuente de algunos de los factores restrictivos que se han identificado en El Salvador. O'Brien y Leichenko describen este juego complejo de fuerzas que afectan a la agricultura como una "doble exposición" (2000) y generará "dobles perdedores" en los sectores afectados. Con respecto a los trabajadores rurales de subsistencia en El Salvador, los cuales juegan un papel pequeño en el contexto de los inminentes CAFTA y ALCA. Basándose en un estudio hecho en México, O'Brien y Leichenko han observado que las estrategias de adaptación al cambio climático en el sector agrícola "pueden verse contrarrestadas o volverse inefectivas debido a las consecuencias asociadas con la globalización económica" (2000: 230). México firmó el Tratado de Libre Comercio de Norte América en 1994 y se considera generalmente que el resultado del mismo fueron las pérdidas experimentadas por parte de la mayoría de pequeños agricultores (Eakin 2005). Este ejemplo puede, por consiguiente, tener implicaciones importantes para El Salvador. De hecho, un proceso que favorezca la adaptación en el sector agrícola tendría que encarar diversos problemas de diferentes orígenes, incluyendo las consecuencias del marco de trabajo del comercio regional y la falta de apoyo a los servicios de extensión agrícola. Las consecuencias del neoliberalismo en América Latina incluyen el aumento de la pobreza y otros impactos sociales, mientras que todavía se tienen que poner en evidencia los beneficios del ajuste estructural. Si no hay perspectivas de crecimiento en el mercado, o subsidios para los agricultores a pequeña escala y no hay un refuerzo de las unidades de apoyo técnico, el panorama de este sector sensible al clima no es alentador. Las consecuencias de la ausencia de dichos factores se verán ampliadas en virtud de la globalización, donde los productos más baratos de los países vecinos serán más asequibles a los salvadoreños que los propios. La dificultad que plantea la falta de acceso a los sistemas de riego y los tipos de cultivo inapropiados puede empeorarse debido al cambio climático; el que se priorice a nivel nacional en el trabajo y las exportaciones no agrícolas no hará más simple ni motivará el acceso a 
mejores sistemas de riego para los productores locales de cultivos de subsistencia. Es, por lo tanto, incierto que tenga éxito tratar de enfrentar los impactos del cambio climático antes de encarar los otros factores.

Estos hallazgos también suscitan preguntas acerca de la importancia de las instituciones sociales a la hora de determinar la capacidad de adaptación. En el caso del Bajo Lempa, las instituciones sociales se han definido como organizaciones y redes sociales. Los estudios de otras regiones han hallado que las instituciones sociales, incluyendo redes en la India y Nepal (Moench y Dixit 2004) y las estructuras y organizaciones que influyen en la colocación de recursos en Vietnam (Adger 2000) juegan un papel clave para determinar la capacidad adaptativa de las sociedades vulnerables. Se ha observado que las redes sociales informales, que incluyen las relaciones basadas en el parentesco y la amistad, desempeñan un papel en la reducción de la vulnerabilidad en otras sociedades latinoamericanas y las políticas de apoyo tienden a incrementar la participación de los actores locales en la mitigación de desastres (Pelling 2002). Tal como lo notó Alger, no obstante, las instituciones pueden, a la vez, "restringir y facilitar la adaptación al cambio social y ambiental (2000: 654). En El Salvador parece que el papel desempeñado tanto por las instituciones informales como por las formales es, primordialmente, el de apoyo psicológico. La importancia de las organizaciones en la reducción de la vulnerabilidad parece basarse más en la medida en que dichas instituciones son capaces de construir confianza entre los miembros de la comunidad, que en pasos prácticos que se toman en aras de reducir o enfrentar el riesgo. Aunque pueda ser cierto que en El Salvador los actores locales tengan la capacidad de responder a las estructuras sociales que los exponen a riesgos ambientales, las barreras ideológicas están frenando la capitalización de esta capacidad entre la gente que vive en el Bajo Lempa, así como en el país en general. Por consiguiente, el énfasis en construir una capacidad de adaptación sin que se enfrenten $-y$ modifiquenestas restricciones no asegurará la reducción de los riesgos en El Salvador. Más aún, la existencia de instituciones crea una conciencia que deviene en un síndrome de dependencia que podría ser mayor que cualquiera de los aspectos positivos. En El Salvador, esta dependencia representa una barrera considerable para reducir la vulnerabilidad.

A nivel nacional, el papel del SNET no ha sido suficientemente puesto a prueba. Es posible que las estructuras institucionales que se han construido fortalezcan la capacidad de todos los niveles, desde el local al nacional, no sólo para dar respuesta a los riesgos a través de un sistema de alerta temprana más efectivo, sino también para prevenir 
el riesgo a través de la difusión de los resultados de las investigaciones sobre varios aspectos de la hidrología, la meteorología y la sismología en El Salvador. De nuevo, la pregunta sigue siendo si la capacidad de las instituciones será suficiente para reducir la vulnerabilidad a los peligros climáticos, donde los desafíos más dominantes están presentes en las estructuras políticas y sociales. Adger habla acerca de una adaptación institucional, la cual define como "el resultado neto de la evolución de instituciones dentro del medio social más amplio junto a la inercia institucional" (2000: 738). Este autor anota que no sólo los individuos, sino también las instituciones necesitarán adaptarse, refiriéndose en este caso a las instituciones formales, más que las informales. En definitiva, esto parece ser el tipo de ajuste que será necesario poner sobre la mesa en El Salvador -en las estructuras de gobierno y en las instituciones relacionadas con el progreso económico, creencias religiosas, relaciones entre las ONG y otras organizaciones y comunidades locales y el gobierno de El Salvador, y las interpretaciones e implementación de la gestión de riesgo. La herencia de la guerra no se ve afectada tan fácilmente y posiblemente se requiera de tiempo para poder conciliar los diferentes puntos de vista. No parece ser el caso que las barreras de una característica tan superficial como la capacidad de adaptación tendrá como resultado un cambio a largo plazo y sustentable en los niveles de riesgo, en la medida en que esto no afecte las causas medulares de la vulnerabilidad.

En suma, se identifican los factores clave que, en El Salvador, aparecen como obstáculos para adaptarse al cambio y la variabilidad del clima y que, por el contrario, contribuyen a aumentar la vulnerabilidad al cambio climático y que probablemente no se puedan enfrentar fácilmente poniendo énfasis en el fomento de la capacidad de adaptación. Así como esta claro en el concepto de "doble exposición" aplicado en el caso del sector agrícola, los factores mencionados aquí no son simples obstáculos para adaptarse al cambio climático, sino también obstáculos para el desarrollo. Es claro que las diferencias entre las exigencias para el desarrollo y adaptación sustentables son menores.

Sin embargo, esto no implica que la adaptación no sea un concepto útil para guiar las acciones políticas orientadas a enfrentar el riesgo proveniente del cambio climático. Más bien, la adaptación tiene un nicho único y de rápida expansión dentro de los discursos sobre el cambio climático, los riesgos y el desarrollo que posibilitará que las discusiones sobre la adaptación logren una mayor autoridad más allá del proceso existente de políticas globales sobre cambio climático. Las relaciones entre estos 
tres discursos también están ganando atención (PNUD 2004), pero las comunidades intelectuales necesitan fusionarse, de tal manera que las lecciones que se aprenden en una disciplina puedan transmitirse a otras. Parece que una vasta cantidad de literatura que da cuenta de la adaptación al cambio climático es el resultado del discurso sobre el riesgo de peligros. También es evidente que hay un interés dentro de las comunidades intelectuales en compartir su conocimiento entre ellas (PNUD 2002). La evidencia empírica de El Salvador indica que la confluencia entre esos tres campos podría ser necesaria a fin de facilitar la adaptación en la realidad. En esta postura, la adaptación al cambio climático podría verse como un concepto unificador que podría juntar los objetivos de las políticas de reducción de la vulnerabilidad y el riesgo, y promover un desarrollo sustentable.

Traducción: Luis Alvarenga

\section{Bibliografía}

Acevedo, C., Barry D., Rosa, H. (1995) "El Salvador's Agricultural Sector: Macroeconomic Policy, Agrarian Change, and the Environment", World Development 23 (12) 2153-2172.

Adger, N. and Brooks, N. (2003)

"Does Global Environmental Change Cause Vulnerability to
Disaster?", en: Pelling, M. (ed.) Natural Disasters and Development in a Globalising World. Routledge: Londres.

Adger, W. N. (2000) "Institutional Adaptation to Environmental Risk under the Transition in Vietnam", Annals of the Association of American Geographers 90 (4) 738-758.

Bankoff, G. (2001) "Rendering the World Unsafe: 'Vulnerability and Western Discourse', Disasters 25 (1) 19-35.

Bhandari, P. (2003) "Environmental vulnerability: the climate change and sustainable development context", in Environmental threats, vulnerability and adaptation, The Energy and Resources Institute (TERI): Nueva Delhi.

Brocket, C. D. (1990) Land and Poverty - Agrarian Transformation and Political Conflict in Central America. Unwin Hyman: Boston.

Cuéllar, N., Gómez, S., Kandel, S., Rosa, H. (2002) "Rural Poverty and Environment in El Salvador: Lessons for Sustainable Livelihoods", PRISMA: San Salvador.

Sitio de internet de la Dirección General de Protección Civil (2009) www.proteccioncivil.gob.sv 
Eakin, H. (2005) "Institutional Change, Climate Risk, and Rural Vulnerability: Cases from Central Mexico", World Development, 33 (11): 1923-1938.

Jones, P.G. and Thornton, P.K. (2003) "The Potential Impact of Climate Change on Maize Production in Africa and Latin America in 2005", Global Environmental Change, 13 (1) 51-59.

Lavell, A. (2004) "The Lower Lempa River Valley, El Salvador: Risk Reduction and Development Project", in Bankoff, G., Frerks, G., Hilhorst, D. (eds.) Mapping Vulnerability: Disasters, Development and People. Earthscan: Londres.

Liverman, D. M. (1999) "Vulnerability and Adaptation to Drought in Mexico", Natural Resources Journal, 39 (1)99-115.

Medina, R., García, M., de Larios, S., Gómez, I., Cuellar, N., Rosa, H. (2002) Dimensiones ambientales de la vulnerabilidad en EI Salvador: El caso del bajo Lempa, PRISMA: San Salvador.

Moench, M.A. and Dixit. A. (2004) (eds.) Adaptive Capacity and Livelihood Resilience, Institute for Social and Environmental Transition: Nepal y Boulder.

Moisa, B. (1996) "Desastres y relaciones de género en comunidades del bajo Lempa, departa- mento de Usulután" CEPRODE, unidad de Investigaciones: San Salvador.

O'Brien, K. and Leichenko, R. (2000) "Double Exposure: Assessing the Impacts of Climate Change Within the Context of Economic Globalisation", Global Environmental Change, 10 (3) 221-232.

Pelling, M. (2002) "Assessing Urban Vulnerability and Social Adaptation to Risk", International Development Planning Review, 24(2) 59-76.

Pelling, M. (2003) Social Capital Hazards and Adaptation Strategies for the Vulnerable. Research Paper, University of East Anglia, Norwich.

Perla- Alfaro, F. de M. (2006) Sustainability, governance and capacity building in the South: A Conceptual Framework. Documento presentado en la reunión anual de WSC-SD, Rasa, Suiza.

Sitio de internet del SNET (Servicio Nacional de Estudios Territoriales) (2004) http://www.snet. gob.sv/

PNUD (2002) Síntesis de la reunión del grupo de expertos, denominada "Integrar la reducción de desastres con la adaptación al cambio climático", 17-19 de junio de 2002, La Habana, Cuba, PNUD: La Habana. 
PNUD (2004) Reducing Disaster Risk: A Challenge for Development, PNUD: Ginebra.

Walker T. S. and Jhoda N. S. (1986) "How Small Farm Households Adapt to Risk, in Hazell, P., Pomeranda, C. and Valdez A. (eds.) Crop Insurance for Agricultural Development: Issues and Experience, Johns Hopkins University Press; Baltimore.
Wisner, B, Blaikie, P. and Cannon, T. (2004) At Risk: Natural Hazards, People's Vulnerability and Disasters, segunda edición, Routledge: Londres.

Wisner, B. (2001) "Risk and Neoliberal State: Why Post-Mitch Lessons Didn't Reduce El Salvador's Earthquake Losses", Disasters, 25 (3) 251-268. 